\title{
Effect of side-chain asymmetry on the intermolecular structure and order-disorder transition in alkyl-substituted polyfluorenes
}

\author{
Knaapila, Matti; Stepanyan, R.; Torkkeli, M.; Haase, D.; Frohlich, N.; Helfer, A.; Forster, M.; Scherf, U.
}

Published in:

Physical Review E

Link to article, DOI:

10.1103/PhysRevE.93.042504

Publication date:

2016

Document Version

Publisher's PDF, also known as Version of record

Link back to DTU Orbit

Citation (APA):

Knaapila, M., Stepanyan, R., Torkkeli, M., Haase, D., Frohlich, N., Helfer, A., Forster, M., \& Scherf, U. (2016). Effect of side-chain asymmetry on the intermolecular structure and order-disorder transition in alkyl-substituted polyfluorenes. Physical Review E, 93(4), [042504]. https://doi.org/10.1103/PhysRevE.93.042504

\section{General rights}

Copyright and moral rights for the publications made accessible in the public portal are retained by the authors and/or other copyright owners and it is a condition of accessing publications that users recognise and abide by the legal requirements associated with these rights.

- Users may download and print one copy of any publication from the public portal for the purpose of private study or research.

- You may not further distribute the material or use it for any profit-making activity or commercial gain

- You may freely distribute the URL identifying the publication in the public portal 


\title{
Effect of side-chain asymmetry on the intermolecular structure and order-disorder transition in alkyl-substituted polyfluorenes
}

\author{
M. Knaapila, ${ }^{1, *}$ R. Stepanyan, ${ }^{2}$ M. Torkkeli, ${ }^{3}$ D. Haase, ${ }^{4}$ N. Fröhlich, ${ }^{5}$ A. Helfer, ${ }^{5}$ M. Forster, ${ }^{5}$ and U. Scherf ${ }^{5}$ \\ ${ }^{1}$ Department of Physics, Technical University of Denmark, 2800 Kgs. Lyngby, Denmark \\ ${ }^{2}$ Materials Science Centre, DSM Research, 6160 MD Geleen, The Netherlands \\ ${ }^{3}$ Department of Physics, University of Helsinki, 00014 Helsinki, Finland \\ ${ }^{4}$ MAX IV Laboratory, Lund University, 22100 Lund, Sweden \\ ${ }^{5}$ Macromolecular Chemistry Group (buwmakro), Bergische Universität Wuppertal, 42119 Wuppertal, Germany
}

(Received 21 December 2015; published 28 April 2016)

\begin{abstract}
We study relations among the side-chain asymmetry, structure, and order-disorder transition (ODT) in hairy-rod-type poly(9,9-dihexylfluorene) (PF6) with two identical side chains and atactic poly(9-octyl-9-methylfluorene) (PF1-8) with two different side chains per repeat. PF6 and PF1-8 organize into alternating side-chain and backbone layers that transform into an isotropic phase at $T^{\mathrm{ODT}}(\mathrm{PF} 6)$ and $T_{\mathrm{bi}}^{\mathrm{ODT}}(\mathrm{PF} 1-8)$. We interpret polymers in terms of monodisperse and bidisperse brushes and predict scenarios $T^{\mathrm{ODT}}<T_{\mathrm{bi}}^{\mathrm{ODT}}$ and $T^{\mathrm{ODT}} \sim T_{\mathrm{bi}}^{\mathrm{ODT}}$ for high and low grafting densities (the side-chain length above or below the average grafting distance). Calorimetry and x-ray scattering indicate the condition $T^{\mathrm{ODT}}(\mathrm{PF} 6) \sim T_{\mathrm{bi}}^{\mathrm{ODT}}(\mathrm{PF} 1-8)$ following the low grafting prediction. $\mathrm{PF} 6$ side chains coming from the alternating backbone layers appear as two separate layers with thickness $H$ (PF6), whereas PF1-8 side chains appear as an indistinguishable bilayer with a half thickness $H_{\text {bilayer }}(\mathrm{PF} 1-8) / 2 \approx H(\mathrm{PF} 6)$. The low grafting density region is structurally possible but not certain for PF6 and confirmed for PF1-8.
\end{abstract}

DOI: 10.1103/PhysRevE.93.042504

\section{INTRODUCTION}

Hairy-rod polymers consist of a stiff backbone and flexible side chains that organize into alternating backbone and side-chain layers and experience an order-disorder transition (ODT) on heating [1-5]. Phase behavior of alkyl-substituted conjugated polymers can be understood in similar terms, and this was illustrated early with polythiophenes [6,7]. These reports were followed by other conjugated polymers with similar structures and an ODT in what regard alkyl-substituted polyfluorenes (PFs) represent an archetypical example [8-10]. Despite their seemingly simple chemical structure they show a variety of supramolecular structures including a long range period that corresponds to the length of the polymer [11,12]; PF nanotubes [13], and a wrap over single-walled carbon nanotubes (SWCNTs) selective to their diameters [14]. This surprising diversity has made PFs interesting not only from the optoelectronic, but also from the structural point of view.

Most structural reports concern symmetric PFs where each fluorene repeat is grafted by two identical alkyl side chains. Ways to diversify side chains include alternating $[15,16]$ and random copolymers $[17,18]$ and a homopolymer route where one of identical side chains is replaced by another side chain. This has been demonstrated for isotactic poly(9-dodecyl-9methyl-fluorene) that organizes into a hexagonal mesophase [19] and shows properties, such as a selective nanotube wrapping preferring semiconducting over metallic SWCNTs [20]. Yet the phase behavioral studies of asymmetrically substituted PFs remain limited.

A bidisperse polymer brush is defined as a mixture of flexible chains of two lengths bound with one "anchoring" end, which typically means a layer configuration [21-23]. In one

\footnotetext{
*matti.knaapila@fysik.dtu.dk
}

example, a poly(2-vinylpyridine)-polystyrene (P2VP- $b$-PS) block copolymer with a bidisperse PS block was spin coated on the substrate such that the P2VP layer formed an anchor on the substrate [24]. PS formed the film surface, and a selective toluene solvent was used to swell the PS layer to form an open brush. In this system, the chains could be extended or compressed depending on their densities. Similar layers have also been formed using Langmuir troughs which allow quantitative experiments vis-à-vis theoretical studies [25,26]. In another example, flexible bottlebrush polymers with bidisperse side-chain lengths were further incorporated into Langmuir monolayers [27]. Furthermore, the chains may be not only bidisperse but also chemically different, which allows tuning their stretching by solvent quality [28,29]. All these ideas can be further modified by introducing polydisperse polymer brushes [30].

In this paper, we study side-chain asymmetry and hairy-rod polymers. First, we put forward a theory where bidisperse hairy-rod polymers with two different side-chain lengths are understood as bidisperse polymer brushes where rodlike backbone stacks correspond to an anchor layer. This provides predictions for ODT temperatures depending on the sidechain symmetry and grafting density. Second, we study these predictions using poly(9,9-dihexylfluorene) (PF6) with two side chains of equal length and atactic poly(9-octyl-9methyl-fluorene) (PF1-8) with two side chains of different lengths. Both polymers show similar ODT temperatures, and this follows the low grafting prediction. Structural analysis identifies differences in terms of crystallite size and side-chain packing. It confirms the low-density assumption with PF1-8 and can favor it with PF6.

This study implies that alkyl-substituted PFs can be discussed with reference to bidisperse polymer brushes. Beginning with stiff homopolymers, it is also an addition to the copolymer and bottlebrush literature. 


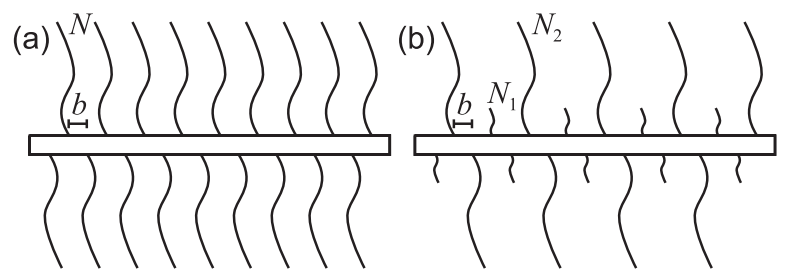

FIG. 1. Side view of hairy-rod polymers with (a) monodisperse or (b) bidisperse side chains. Here $b$ is the grafting distance and $N$, $N_{1}$, and $N_{2}$ are the numbers of side-chain beads with $N_{2} \gg N_{1}$.

\section{THEORY}

\section{A. Hairy-rod polymers with monodisperse or bidisperse side chains}

Figure 1(a) illustrates a hairy-rod polymer with identical equidistantly spaced side chains as detailed in Ref. [4]. These polymers consist of a stiff backbone with diameter $d$ and flexible side-chain "coils" attached on the backbone at a distance $b$ from each other (denoted as a grafting distance). In this consideration each grafting point has one side chain, and the distance $b$ equals the backbone length per one side chain. Each side chain consists of $N$ beads each having a volume $v$. The statistical length of a flexible chain is denoted as $a$, and the size of an unperturbed coil can be estimated from a valence angle model,

$$
R_{c}=a \sqrt{N}
$$

where

$$
a=a_{0} \sqrt{\frac{1-\cos \gamma^{\prime}}{1+\cos \gamma^{\prime}}} \approx 2.2 \AA
$$

with the bond length of $a_{0}=1.54 \AA$ and the bond angle of $\gamma^{\prime}=109.5^{\circ}$. We have previously shown that this model is a powerful starting point when predicting solid state phases of PF polymers [31].

Figure 1(b) illustrates a hairy-rod polymer with two different equidistantly placed side chains with the lengths $N_{1}$ and $N_{2}$ with $N_{2} \gg N_{1}$. Following the notation used for bidisperse polymer brushes [21-23], we denote discussed hairy-rod side chains as monodisperse and bidisperse.

Hairy-rod polymers can order into a variety of microphases that experience an ODT to the disordered phase on heating. These microphases include a lamellar structure which resembles a lamellar phase of block copolymers as schematically illustrated in Figs. 2 and 3. These schemes differentiate high

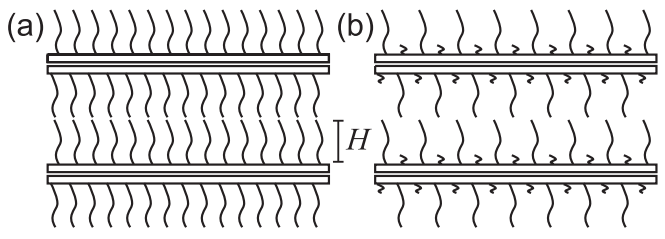

FIG. 2. Side view of hairy-rod layers with high grafting density and (a) monodisperse or (b) bidisperse side-chain length. These structures correspond to end-to-end side-chain packing. $H$ is the thickness of the side-chain layer and typically $b<H$.

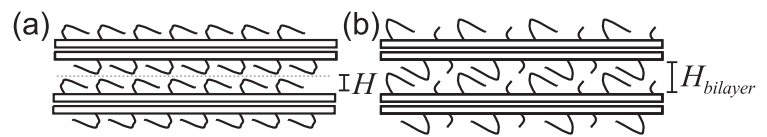

FIG. 3. Side view of hairy-rod layers with low grafting density and (a) monodisperse or (b) bidisperse side-chain length. These structures involve nearly touching main chains. $H$ is the thickness of the side-chain layer and typically $b>H$ and $H_{\text {bilayer }}=2 H$.

and low grafting density regimes which correspond to long or short side chains.

\section{B. High grafting density}

We first consider hairy-rod polymers in the high grafting density regime [4]. This means that the condition $b<a \sqrt{N}$ is fulfilled for densely grafted side chains with $N \gg 1$. In this dense brush regime, the side chains decrease their end-to-end distance as illustrated for the lamellar microstructure in Fig. 2. However, our analysis is also applicable for other ordered microphases, such as a hexagonal phase.

The ODT can be estimated by writing the free energies of ordered and disordered phases and setting them equal at the transition point. For the ordered phase, the free energy per segment $b$ can be expressed as

$$
F^{(\text {ord })} \simeq \gamma b d+\frac{3}{2} \frac{H^{2}}{N a^{2}} k_{B} T
$$

where the first contribution corresponds to the rod-coil interaction energy and the second contribution to the entropic part of the free energy due to the side-chain stretch. Here $\gamma$ is an interfacial energy parameter, $k_{B}$ is the Boltzmann constant, and $T$ is temperature. The thickness for the side-chain layer $H$ is obtained from the incompressibility condition for the side chains and reads as

$$
H=\frac{v N}{b d} .
$$

For the disordered phase, the free energy per segment corresponds to the interfacial free energy as

$$
F^{(\text {dis })} \simeq 2 \gamma b d
$$

which is similar to the corresponding term in Eq. (3) but twice as high because the contact area is doubled. When the free energies are set equal, we obtain an estimation for the ODT temperature $T^{*}$ as

$$
k_{B} T^{*} \sim \frac{2 \gamma}{3}\left(\frac{a}{v}\right)^{2} \frac{(b d)^{3}}{N} .
$$

The approach described above can be generalized to the bidispersed case by addressing the elastic contribution from the bidisperse brush of the side chains following the ideas detailed in Ref. [21]. The elastic free energy of the dense bidisperse brush reads as

$$
F_{e l} \simeq \frac{3}{2}\left[\frac{v}{a b d}\right]^{2}\left[N_{1}+\frac{N_{2}-N_{1}}{8}\right] k_{B} T,
$$


which leads to the modification of Eq. (6) and the ODT temperature $T_{\mathrm{bi}}^{*}$ as

$$
k_{B} T_{\mathrm{bi}}^{*} \sim \frac{2 \gamma}{3}\left(\frac{a}{v}\right)^{2} \frac{(b d)^{3}}{N_{1}+\left(N_{2}-N_{1}\right) / 8}
$$

\section{Low grafting density}

We consider next the low grafting density regime illustrated in Fig. 3. This means that the condition $b>a \sqrt{N}$ is fulfilled and short or distant chains interact weakly. The side chains become compressed rather than stretched and are likely to extend in the direction perpendicular to the layers on the plane of the polymer backbones approximately to the distance $l \sim$ $\sqrt{b d}$. is

The elastic free energy associated with this conformation

$$
F_{\mathrm{el}} \simeq \frac{l^{2}}{N a^{2}} k_{B} T
$$

Analogy to the brush regime yields the ODT temperature $T^{* *}$ as

$$
k_{B} T^{* *} \sim \gamma N a^{2} .
$$

Similarly, we can follow the above derivation for the polymers with bidisperse side chains assuming the condition $N_{2} \gg N_{1}$ where the role of shorter side chains becomes negligible. From this we find that the ODT temperature $T_{\mathrm{bi}}^{* *}$ fulfills a condition,

$$
k_{B} T_{\mathrm{bi}}^{* *} \sim \gamma N_{2} a^{2}
$$

\section{EXPERIMENT}

\section{A. Materials}

Figure 4 shows the chemical structures of the employed polymers. PF6 is known from the prior literature whereas PF1-8 is novel. PF6 with the number average molecular weight $\left(M_{\mathrm{n}}\right)$ of $84 \mathrm{~kg} / \mathrm{mol}$ and the weight average molecular weight $\left(M_{\mathrm{w}}\right)$ of $200 \mathrm{~kg} / \mathrm{mol}$ was prepared following the Yamamoto-type polycondensation procedure with the corresponding 2,7-dibromo-9,9-dihexylfluorene as a monomer and bis(1,5-cyclooctadiene)nickel(0) as a

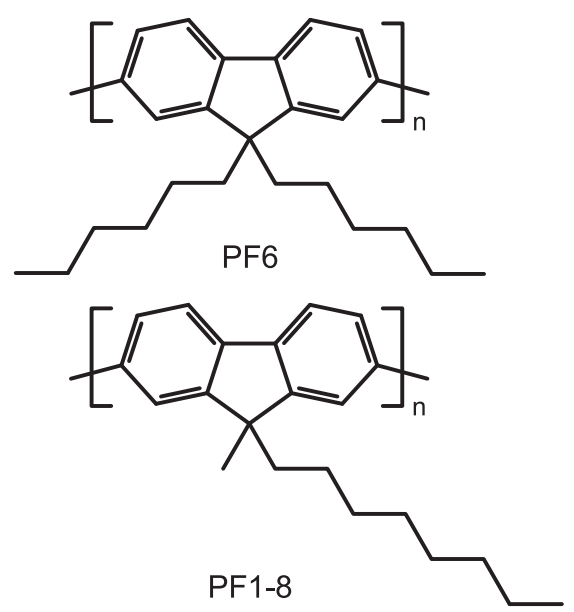

FIG. 4. Chemical structures of PF6 and PF1-8. coupling agent as described in Ref. [32]. Atactic PF18 with $M_{\mathrm{n}}=26 \mathrm{~kg} / \mathrm{mol}$ and $M_{w}=33 \mathrm{~kg} / \mathrm{mol}$ was prepared by Suzuki-type aryl-aryl cross coupling of the $A B$ type monomer /rac/-(2-bromo-9-octyl-9-methylfluorene)-7(4,4,5,5-tetramethyl-1,3,2-dioxaborolane) following the ideas in Ref. [33]. After coupling, both polymers were purified by solvent extraction with methanol, acetone, ethyl, acetate, and chloroform. The chemical structures were confirmed by ${ }^{1} \mathrm{H}$ and

${ }^{13} \mathrm{C}$ NMR spectroscopy and the molecular weights determined by gel permeation chromatography using narrowly distributed polystyrene standards.

With PF1-8 we obtained: ${ }^{1} \mathrm{H}-\mathrm{NMR}\left(600 \mathrm{MHz}, \mathrm{CDCl}_{3}\right.$, $305 \mathrm{~K}): \delta(\mathrm{ppm})=7,90 \quad(d, J=7,9 \mathrm{~Hz}) ; 7,79-7,69 \quad(\mathrm{~m})$; 2,29-2,08 (m); 1,68 (s); 1,32-1,05 (m); 0,99-0,78 ppm (m).

${ }^{13} \mathrm{C}\left\{{ }^{1} \mathrm{H}\right\}-\mathrm{NMR}\left(151 \mathrm{MHz}, \mathrm{CDCl}_{3}, 305 \mathrm{~K}\right): \delta(\mathrm{ppm})=153,2$; 140,$7 ; 139,1 ; 126,3 ; 121,5 ; 120,2 ; 51,1 ; 40,8 ; 31,8 ; 30,0$; 29,$7 ; 29,2 ; 27,0 ; 24,4 ; 22,6 ; 14,1 \mathrm{ppm}$. Gel permeation chromatography (tetrahydrofuran): chloroform fraction: $M_{n}=25.5 \mathrm{~kg} / \mathrm{mol}, M_{w}=33 \mathrm{~kg} / \mathrm{mol}$, polydispersity $=$ 1.29. UV $\left(\mathrm{CHCl}_{3}\right) \lambda=388 \mathrm{~nm}$. UV/vis (film) $\lambda=382$ nm. PL $\left(\mathrm{CHCl}_{3}, \lambda_{\mathrm{ex}}=350 \mathrm{~nm}\right) 414,437,475 \mathrm{~nm}$. PL (film, $\left.\lambda_{\text {ex }}=350 \mathrm{~nm}\right)=429,451,490 \mathrm{~nm}$.

Bulk polymers were studied using differential scanning calorimetry (DSC) and x-ray diffraction (XRD). For DSC measurements, polymers were used as is. For XRD measurements, both polymers were melted directly to the sample holder. This means that the measured first heating represents the second heating of the as-received sample.

\section{B. Methods}

The DSC curves were measured using a DSC1 STAR system from Mettler Toledo under an argon flow. The measurement protocol contained the first heating from the room temperature, cooling back to the room temperature, and heating up again. The heating and cooling rates were $3{ }^{\circ} \mathrm{C} / \mathrm{min}$. The sample size was 4-8 mg.

XRD experiments were carried out at the I711 Beamline at MAX IV Laboratory in Lund (Sweden). The beam was monochromatized using an asymmetrically cut single Si(111) monochromator focused on the sample. The x-ray energy was $12.5 \mathrm{keV}$, and the beam size was $0.5 \times 0.5 \mathrm{~mm}^{2}$. Diffraction patterns were measured in the transmission geometry using a Titan CCD with $165 \mathrm{~mm}$ diameter (Oxford Diffraction). The samples were $2 \mathrm{~mm}$ wide and $1 \mathrm{~mm}$ thick blocks melted in a sample holder that was custom made from copper. The temperature was controlled using a Huber HTC 9634 high temperature device directly connected to the sample holder and monitored by an external thermocouple connected to the holder. The heating and cooling rates were $\sim 3{ }^{\circ} \mathrm{C} / \mathrm{min}$. The sample sizes were a few milligrams corresponding to those used in DSC measurements.

\section{RESULTS AND DISCUSSION}

\section{A. ODT}

Using the framework presented in the theoretical section, we can first identify two possible scenarios for the relation between hairy-rod ODT and side-chain asymmetry. 
In the high grafting density regime, we compare Eqs. (6) and (8) and assume that $N_{1} \ll N_{2}$. This indicates that $T^{*}$ is similar to $T_{\text {bi }}^{*}$ with $N=N_{2} / 8$. This relation can be understood against the fact that the limiting case $N_{1} \ll N_{2}$ is similar to the reduction of the grafting density by a factor of 2 , i.e., doubling of the distance $b$. As the transition temperature increases as $b^{3}$, the factor of 2 shift in $b$ results in a temperature shift by a factor of 8. Therefore, if $N$ and $N_{2}$ are on the same order of magnitude, $T^{*}$ should be lower than $T_{\mathrm{bi}}^{*}$,

$$
T^{*}<T_{\mathrm{bi}}^{*} \text {. }
$$

In the low grafting density regime, we compare Eqs. (10) and (11) and assume that $N_{1} \ll N_{2}$. From this follows that the transition temperature $T^{* *}$ for the monodisperse case should be similar to $T_{\mathrm{bi}}^{* *}$ for the bidisperse case with $N \approx N_{2}$ :

$$
T^{* *} \approx T_{\mathrm{bi}}^{* *} .
$$

Although the grafting density regime is defined by $b$, the transition temperatures for the low grafting density do not depend on $b$. This agrees with the idea of weakly interacting side chains.

We next follow theoretical reasoning and consider experimental transition temperatures for PF6 and PF1-8. First, we assume that $6 \approx 8$ and thus the condition $N \approx N_{2}$ holds for the given polymer pair. Furthermore, we assume that $1 \ll 8$ and thus the condition $N_{1} \ll N_{2}$ holds for PF1-8. This allows us to hypothesize that the studied polymers follow Eq. (13).

Solid state phases of PF6 include the monoclinic $\alpha$ phase and the coexisting triclinic $\alpha^{\prime}$ phase. The latter represents a minority phase as detailed by Chen and co-workers [34]. When heated, PF6 shows subsequent phase transitions from the coexisting $\alpha^{\prime}$ phase to the pure $\alpha$ phase and from the pure $\alpha$ phase to a nematic phase. Crystalline $\alpha$ and $\alpha^{\prime}$ phases are structurally close and agree with our simplified idea of lamellae whereas the nematic phase agrees with our idea of disordered melt. This means that PF6 corresponds to our idea of a hairyrod polymer with symmetric monodisperse side chains. The transition from the $\alpha$ phase to the nematic phase corresponds to our notation of ODT with the transition temperature $T^{* *}(\mathrm{PF} 6)$. PF6 is polymorphic and shows a mesomorphic $\beta$ phase that exists in as-cast films and transmutes to the $\alpha$ phase on heating. Yet the amount of $\beta$ phase is always small and can be ignored in our considerations. The structure and phase behavior of PF1-8 are reported here.

Figure 5 shows DSC curves of PF6 and PF1-8 measured with the heating and cooling rates of $3{ }^{\circ} \mathrm{C} / \mathrm{min}$. The heating curve represents the second heating of the as-received sample and preceded the shown cooling curve.

PF6 shows sharp peaks at $254^{\circ} \mathrm{C}$ and $211^{\circ} \mathrm{C}$ on heating and cooling which agree almost exactly with the previously reported peaks at $250^{\circ} \mathrm{C}$ and $210^{\circ} \mathrm{C}$ on heating and cooling with the rate of $5^{\circ} \mathrm{C} / \mathrm{min}$ [34]. Following the previous interpretation of Chen et al. [34], we classify these peaks as transitions between the $\alpha$ phase and the nematic phase corresponding to our notation $T^{* *}(\mathrm{PF} 6)$. With heating the sample shows a slight shift at about $100^{\circ} \mathrm{C}-110^{\circ} \mathrm{C}$ which seems to agree with the previously reported glass transition temperature $T_{g}$ at $103{ }^{\circ} \mathrm{C}$ [34]. There is also a broad feature at $195^{\circ} \mathrm{C}-215^{\circ} \mathrm{C}$ on heating and at $120^{\circ} \mathrm{C}-140^{\circ} \mathrm{C}$ on cooling. Since we plot the second heating of the as-received sample,

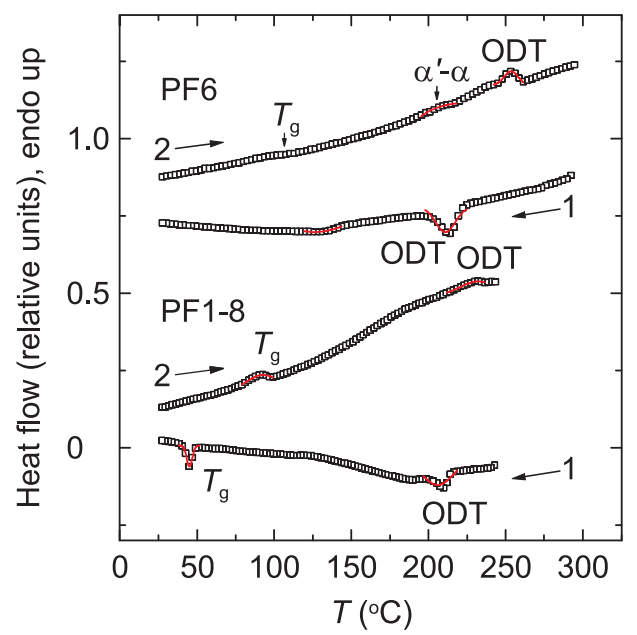

FIG. 5. DSC curves of PF6 and PF1-8 (open symbols) with fits to the peaks (red solid lines). The heating and cooling rates were $3{ }^{\circ} \mathrm{C} / \mathrm{min}$, and the heating represents the second heating of the asreceived sample. The horizontal arrows show the direction and order of the cycle. The curves are shifted for clarity.

the first feature may be identified as the transition from the $\alpha^{\prime}$ phase to the $\alpha$ phase as reported for the second heating in Ref. [34].

PF1-8 shows similar peaks at $232{ }^{\circ} \mathrm{C}$ and $206^{\circ} \mathrm{C}$ which we interpret as ODT with the transition temperature $T_{\mathrm{bi}}^{* *}(\mathrm{PF} 1-8)$. The data also show sharp peaks at $93^{\circ} \mathrm{C}$ and $45^{\circ} \mathrm{C}$ which are likely associated with the glass transition.

Figure 6 shows the XRD patterns of PF6 and PF1-8 during a heating-cooling cycle where the sample size and heating and cooling rates correspond to the DSC measurements. The measurements were performed after annealing and thus the heating represents the second heating of as-received samples also corresponding to the DSC measurements shown in Fig. 5. For clarity reasons we show only every third measured XRD curve, but the data analysis was performed with the full data set.

PF6 shows sharp reflections that can be indexed following the known $\alpha$ and $\alpha^{\prime}$ phases [34]. PF1-8 shows similar but not identical reflections that allow indexation according to the tentatively orthorhombic structure (vide infra). Both structures are approximately lamellar corresponding to the idea shown in Figs. 2 and 3. For both polymers, these sharp reflections turn to broad features on heating and come back upon cooling with clearly observable hysteresis. We attribute these changes to the ODTs from the ordered lamellar to the disordered melt marking the transition temperatures.

The transition temperatures determined by both DSC and XRD are compiled in Table I. The values deduced from DSC agree well with the ones determined by XRD. Characteristic for polymers, these transitions show significant hysteresis and are higher on heating. The transitions of PF6 are somewhat higher than those of PF1-8 on heating but similar on cooling. No evidence is found for the high grafting scenario, i.e., $T^{*}(\mathrm{PF} 6)<T_{\mathrm{bi}}^{*}(\mathrm{PF} 1-8)$, but the data rather indicate that polymers follow the low grafting scenario, i.e., $T^{* *}(\mathrm{PF} 6) \approx$ $T_{\mathrm{bi}}^{* *}(\mathrm{PF} 1-8)$. Even though thermal experiments can be interpreted against theoretical predictions, they cannot confirm 

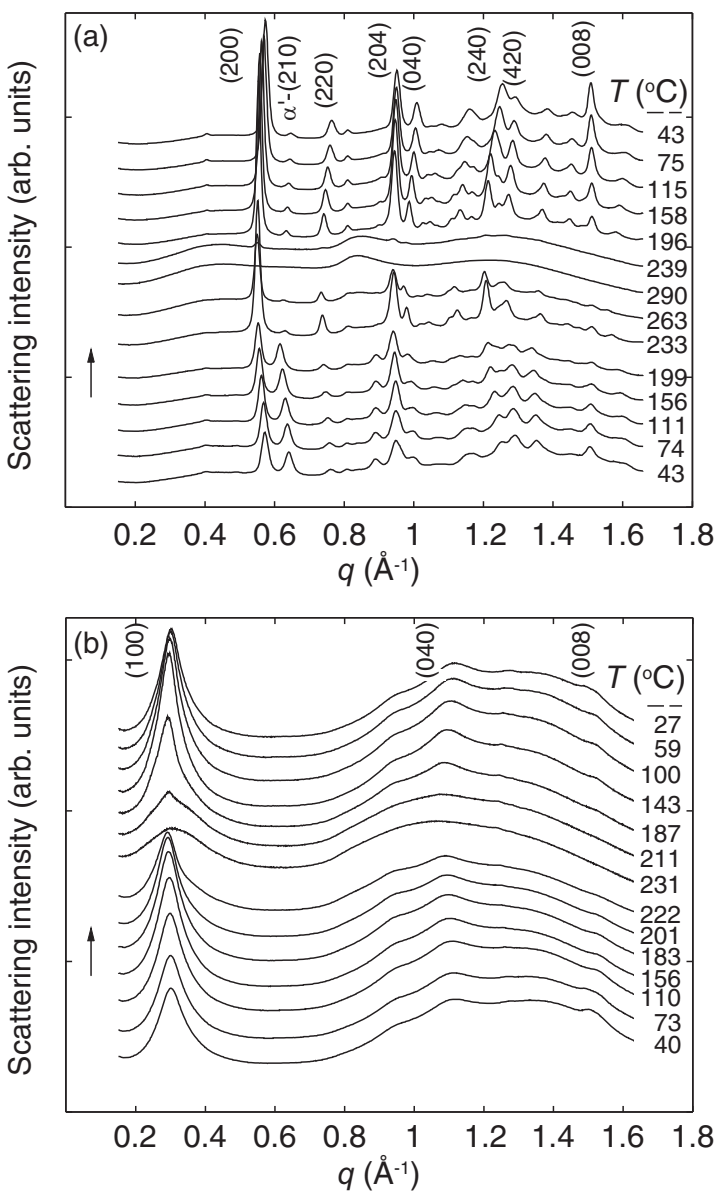

FIG. 6. XRD patterns of annealed (a) PF6 and (b) PF1-8 as a function of temperature. The heating and cooling rates were $3{ }^{\circ} \mathrm{C} / \mathrm{min}$. The curves are integrated from two-dimensional data and staggered for clarity. The indices are discussed in Sec. IV B (vide infra).

structural assumptions for layers and grafting densities. For this reason, we proceed to further structural investigations.

\section{B. Structural analysis}

Figure 6 provides the first idea of layer structure coming from the integrated XRD patterns. PF6 layers are manifestations of the monoclinic $\alpha$ phase with the lattice parameters $\tilde{a}(\mathrm{PF} 6)=21.5 \AA, \tilde{b}(\mathrm{PF} 6)=24.7 \AA, \tilde{c}(\mathrm{PF} 6)=33.2 \AA$, and $\gamma=96^{\circ}$ [34]. The indexation in Fig. 6(a) follows this idea, and the main reflections (200), (040), and (008) describe the lamellar spacing, stacking between polymer backbones, and

TABLE I. Transition temperatures for PF6 and PF1-8 during a heating-cooling cycle with the heating and cooling rate of $3^{\circ} \mathrm{C} / \mathrm{min}$.

\begin{tabular}{lccc}
\hline \hline Cycle direction & Method & $T^{* *}(\mathrm{PF} 6)\left({ }^{\circ} \mathrm{C}\right)$ & $T_{\mathrm{bi}}^{* *}(\mathrm{PF} 1-8)\left({ }^{\circ} \mathrm{C}\right)$ \\
\hline Heating & DSC & $254 \pm 3$ & $232 \pm 6$ \\
& XRD & $276 \pm 13$ & $227 \pm 5$ \\
Cooling & DSC & $211 \pm 4$ & $206 \pm 6$ \\
& XRD & $218 \pm 20$ & $197 \pm 8$ \\
\hline \hline
\end{tabular}

half of the polymer repeat. (We note that here the stacking does not need to mean strong $\pi-\pi$ interactions between aromatic groups.) Furthermore, we can identify the reflection (210) that belongs to the minority phase $\alpha^{\prime}$. The data indicate that side chains coming from opposite backbone layers are segregated rather than mixed or interdigitated (but this does not necessarily mean that they are stretched). The thickness of the side-chain layer is $H(\mathrm{PF} 6) \approx 5.6 \AA$.

PF1-8 shows fewer and broader peaks than PF6 and is clearly less crystalline with a smaller crystallite size. This polymer appears still lamellar, and in Fig. 6(b) we can identify reflections (040) and (008) matching their equivalents for PF6. This gives an idea of lattice parameters on the $b c$ plane and allows us to estimate the stacking distance $\tilde{b}(\mathrm{PF} 1-8) / 4=5.8 \AA$ and the length of repeat unit $\tilde{c}(\mathrm{PF} 1-8) / 4=8.2 \AA$. The fact that the structures on the $b c$ plane are similar justifies a comparison between PF6 and PF1-8. This also means that the backbone layer can be understood as a similar rigid anchor layer as in the case of bidisperse brushes. In contrast, the first $(h 00)$ reflection is at significantly lower scattering angles $\left(q \sim 0.3 \AA^{-1}\right)$ as expected from PF6 analogy. We index this peak as (100) and not as (200).

Like all hairy-rod type polymers, PF1-8 becomes easily aligned by heating and drawing and even spontaneously by mere heating between $T_{g}$ and ODT. This alignment phenomenon allows us to identify crystallographic directions for observed x-ray reflections.

Figure 7 shows the XRD curves of aligned PF1-8 in equatorial and meridional directions (i.e., perpendicular and parallel to the polymer main chain axis c) at $143^{\circ} \mathrm{C}$. Reflections (100) and (040) are distinctive in the equatorial direction while (008) corresponding to the half of the repeat unit is observed in the meridional direction. This directionality confirms that the main chain indexing can be analogous to PF6. We interpret that the reflection (100) does not stem from the polymer layer but from the polymer double layer which agrees with the lamellar period $H_{\text {total }}(\mathrm{PF} 1-8) \approx 21.3 \AA$. Also two further reflections are visible in the meridional direction. If we fix the index (008), these reflections cannot be its multiples in the $00 c$ direction but can be indexed as (104) and (024). This means that the

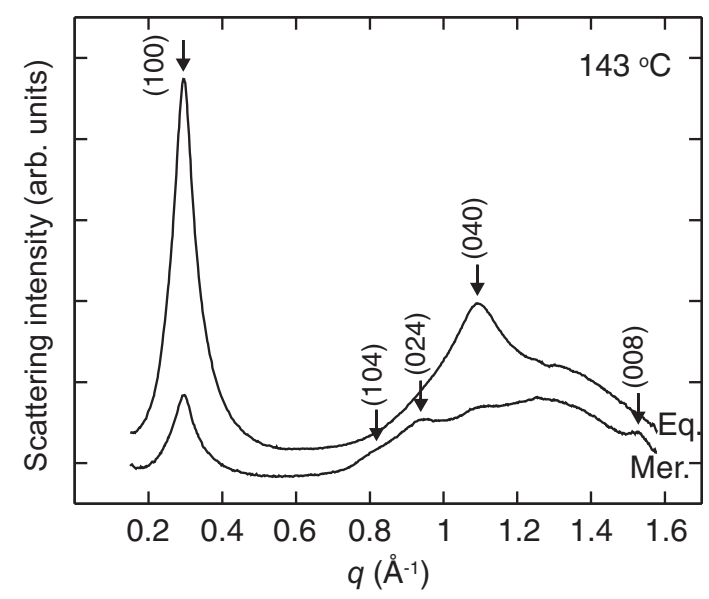

FIG. 7. Equatorial and meridional slice integrals from the twodimensional XRD pattern of aligned PF1-8 at $143 \pm 3{ }^{\circ} \mathrm{C}$. 


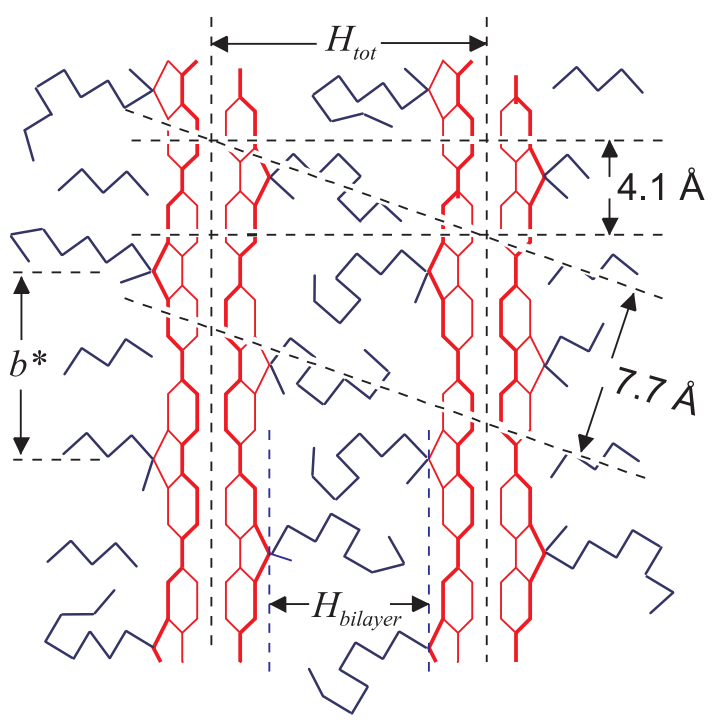

FIG. 8. Schematic of the PF1-8 bilayer structure deduced from the data shown in Fig. 7. The structure on the $a c$ plane is based on the observed reflections and indicates mixing or interdigitation of side chains. $H_{\text {total }}$ refers to the layer spacing and $b^{*}$ to the distance between grafting points. $H_{\text {bilayer }}$ is the thickness of the mixed side-chain layer.

material shows a clear three-dimensional order even if weaker than PF6.

Figure 8 illustrates the proposed PF1-8 structure with the side-chain bilayer. In this structure, the side chains from the one backbone layer are mixed with the side chains from the opposite layer. Although the scheme illustrates them as interdigitated, the side chains remain amorphous while mixed, and we cannot confirm or reject their interdigitation. Furthermore, PF1-8 is atactic, and the side chains have no regular directionality except outwards from the main chain layer.

The thickness of the side-chain bilayer can be estimated from its volume fraction as

$$
H_{\text {bilayer }}=\varphi_{\mathrm{sc}} H_{\text {total }}=\frac{M_{\mathrm{sc}} / \rho_{\mathrm{sc}}}{M_{\mathrm{mc}} / \rho_{\mathrm{mc}}+M_{\mathrm{sc}} / \rho_{\mathrm{sc}}} H_{\text {total }},
$$

where $\varphi_{\mathrm{sc}}$ is the volume fraction of the side chains and $H_{\text {total }}$ is the total lamellar period including side- and main-chain layers. $M_{\mathrm{sc}}$ and $M_{\mathrm{mc}}$ are the molecular weights of side and main chains per monomer, and $\rho_{\mathrm{sc}}$ and $\rho_{\mathrm{mc}}$ are their densities.

This result may be compared to the alternative estimation that accounts for the area of monomer on the $b c$ plane. The side-chain volume per monomer is

$$
V_{\mathrm{sc}}=A_{\text {monomer }} H=\frac{M_{\mathrm{sc}}}{\rho_{\mathrm{sc}} N_{A}},
$$

where $A_{\text {monomer }}$ is the area of monomer on the $b c$ plane, $H$ is the thickness of the side-chain layer, and $N_{A}$ is the Avogadro number. This gives us the thickness of the bilayer as

$$
H_{\text {bilayer }}=2 \frac{M_{\mathrm{sc}}}{A_{\text {monomer }} \rho_{\mathrm{sc}} N_{A}} .
$$

Unit cell parameters and the cell volumes for linear side-chain PFs with increasing side-chain length are listed in Ref. [35]. When assuming constant volume for the main chain we can estimate densities separately for main chain $\rho_{\mathrm{mc}}=1.118 \mathrm{~g} / \mathrm{cm}^{3}$ and side chains $\rho_{\mathrm{sc}}=0.917 \mathrm{~g} / \mathrm{cm}^{3}$. On the other hand, crystallographic data shown provide the area of monomer $A_{\text {monomer }} \approx 47.6 \AA^{2}$ defined by the stacking distance and polymer repeat $\left(5.8 \times 8.2 \AA^{2}\right)$. Using these results Eqs. (14) and (16) give us estimations $H_{\text {bilayer }}(\mathrm{PF} 1-8) \approx$ $10.45 \AA$ and $H_{\text {bilayer }}(\mathrm{PF} 1-8) \approx 9.8 \AA$.

Side chains from one backbone layer represent half of the bilayer volume, and the thickness of the "effective side-chain layer" corresponding to this volume is half of the bilayer thickness, $H(\mathrm{PF} 1-8)=H_{\text {bilayer }}(\mathrm{PF} 1-8) / 2 \approx 5.2 \AA$.

This consideration indicates that apart from side-chain mixing, PF1-8 fits well the structural idea of symmetrically substituted PFs. Separated side-chain layers can be accounted for by the theoretical predictions for both the high and low grafting density regimes [Fig. 2 and Fig. 3(a)]. In contrast, mixed side chains point to the low grafting density regime [Fig. 3(b)].

\section{Analysis of grafting density}

The grafting density regimes can be discussed in terms of grafting distance $b$ compared to the length of unperturbed chain $R_{c}$ and the thickness of side-chain layer $H$. For the high grafting density regime, the condition $b<R_{c}$ implies that the chains are stretched. If the structure is lamellar, the thickness of the side-chain layer is virtually higher than the size of unperturbed side chain $R_{c}<H$ and thus $b<H$. Similarly, for the low grafting density regime, the condition $b>R_{c}$ favors a situation where the layer thickness can be smaller than the size of the side chain, i.e., $R_{c}>H$ and $b>H$.

In the theoretical section, we assume one side chain per grafting point, and thus, the distance between grafting points equals the backbone length per side chain. For the experimental consideration, we define an experimental grafting distance $b^{*}$ as the backbone length per grafting point that can incorporate several side chains. For the discussed polymers this length corresponds to the length of polymer repeat and can be determined from structural data. Along the $\mathbf{c}$ axis, the polymers assume periodicity of four monomers manifested by lattice parameters $\tilde{c}(\mathrm{PF} 6)=33.2 \AA$ and $\tilde{c}(\mathrm{PF} 1-8) \approx 32.8 \AA$. The sizes of polymer repeats and, thus, the experimental distances between grafting points can be estimated from this periodicity as $b^{*}(\mathrm{PF} 6)=\tilde{c}(\mathrm{PF} 6) / 4=8.3 \AA$ and $b^{*}(\mathrm{PF} 1-8)=\tilde{c}(\mathrm{PF} 1-8) / 4=$ $8.2 \AA$. Since PF6 has two side chains per grafting point, we assume that the backbone length per side chain (corresponding to the theoretical definition of grafting distance $b$ ) is half of this, i.e., $b(\mathrm{PF} 6)=b^{*}(\mathrm{PF} 6) / 2 \approx 4.2 \AA$. Since $\mathrm{PF} 1-8$ has only one significant (octyl) side chain per grafting point, we assume that the backbone length per side chain equals the distance between grafting points, i.e., $b(\mathrm{PF} 1-8)=b^{*}(\mathrm{PF} 1-8) \approx 8.2 \AA$.

Two side chains per grafting point mean that $b$ (PF6) should be understood as an average quantity along the long polymer chain. Furthermore, as PF1-8 is atactic, the experimental grafting distance will fluctuate along the polymer chain and can be only expressed as an average density. The average nature of the grafting distance does not violate our theoretical consideration that relies on mean field ideas rather than chemically detailed molecular models. Thus we assume that 
TABLE II. Structural parameters determining the grafting density regime.

\begin{tabular}{lccccc}
\hline \hline & $b^{*}(\AA)$ & $b(\AA)$ & $H_{\text {bilayer }}(\AA)$ & $H(\AA)$ & $R_{c}(\AA)$ \\
\hline PF6 & 8.3 & 4.2 & & 5.6 & 5.4 \\
PF1-8 & 8.2 & 8.2 & 10.5 & 5.2 & 6.2 \\
\hline \hline
\end{tabular}

the molecule stereochemistry does not alter our semiqualitative reasoning.

Experimentally determined parameters $b^{*}, b, H_{\text {bilayer }}$, and $H$ as well as $R_{c}$ values calculated using Eq. (1) are listed in Table II. For PF6 we get $b$ (PF6) $<R_{c}$ (PF6) and $b$ (PF6) $<$ $H$ (PF6), which point in the direction of a brush regime and an arrangement shown in Fig. 2(a). Yet all values are rather similar $H(\mathrm{PF} 6) \approx b(\mathrm{PF} 6) \approx R_{c}(\mathrm{PF} 6)$, and, recalling the separation of side-chain layers, the structure shown in Fig. 3(a) is also possible. For PF1-8, we get $b(\mathrm{PF} 1-8)>R_{c}(\mathrm{PF} 1-8)$ and $b(\mathrm{PF} 1-8)>H(\mathrm{PF} 1-8)$, which point to the low grafting density regime and the situation shown in Fig. 3(b). The observation $H(\mathrm{PF} 1-8) \approx H(\mathrm{PF} 6)$ implies that the comparison between polymers is relevant and can be reduced down to the side-chain symmetry.

\section{Comparison to other PFs}

Comparison of XRD data can be expanded from PF6 to symmetrically substituted poly(9,9-diheptylfluorene) (PF7), poly(9,9-dioctylfluorene) (PF8), poly(9,9-dinonylfluorene) (PF9), and poly(9,9-didecylfluorene) (PF10) discussed in Ref. [35]. In this comparison, the crystalline structure of PF1-8 is weaker as evidenced by fewer and broader reflections. The reflections pertaining to backbone stacking and repeat units are observed and confirm that PF1-8 follows the general scheme of lamellar alkyl-substituted PFs. Contrary to this idea, the first reflection associated with the lamellar spacing is at a significantly smaller angle and indicates a doubled period (or bilayer) with respect to symmetrically substituted PFs.

Figure 9 plots $H$ as a function of side-chain weight for listed PFs. Also shown are the grafting distance $b$ estimated as backbone length per long(er) side chain for PF6 and PF1-8. The size of an unperturbed coil $R_{c}$ is show for comparison.

For PF6-PF9, $H$ is calculated from the $\alpha$ crystalline unit cell parameters $\tilde{b}$ and $\tilde{c}$ and assumed side-chain density $0.95 \mathrm{~g} / \mathrm{cm}^{3}$ listed in Ref. [35]. For PF10, the estimation is based on the mesoscopic chain-to-chain order. The thick solid line gives the expected $H$ that follows a volume-based argument hinging on a side-chain monolayer and a rigid backbone stacking structure on the $b c$ plane. The data show that $H$ increases with increasing side-chain weight and thus with increasing $N$ whereas $R_{c}$ increases as $\sqrt{N}$. The former increase is nearly linear with a curious but statistically significant odd-even effect. Consideration of side-chain weight does not depend on the side-chain symmetry and allows the same argument regardless of the substitution symmetry.

\section{CONCLUSIONS}

We rationalized PF ODT from the lamellar to the isotropic phase in terms of side-chain asymmetry. Hairy-rod-type poly-

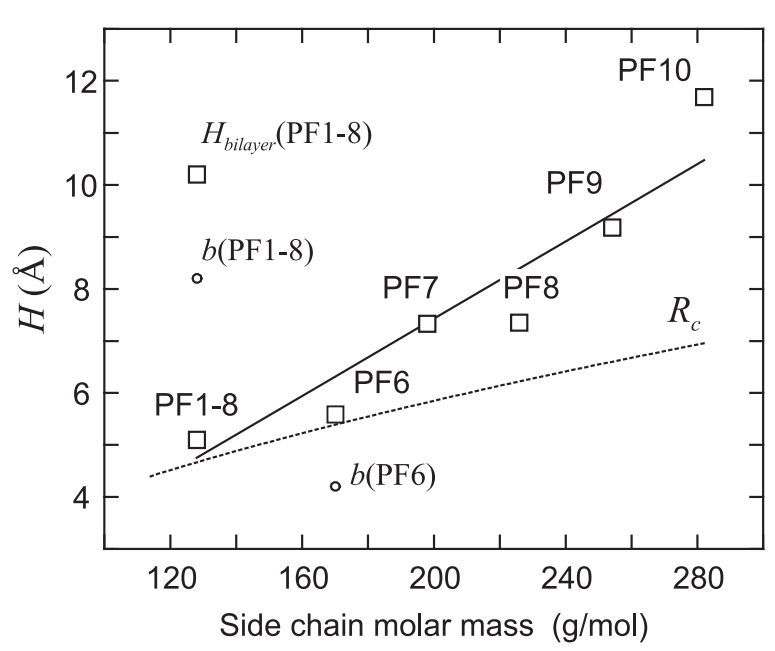

FIG. 9. Experimentally determined $H$ for various PFs (open squares), $H_{\text {bilayer }}$ for PF1-8 (a square in parentheses), and grafting distance $b$ as the backbone length per long(er) side chains for PF6 and PF1-8 (small circles). The solid line is the theoretical $H$ calculated from the volume of the side-chain layer assuming identical unit cell sizes on the $a b$ plane. The dotted line marks the size of an unperturbed coil $R_{c}$.

mers with identical and nonidentical side chains and side-chain lengths $N, N_{1}$, and $N_{2}$ were understood as monodisperse and bidisperse polymer brushes where stacked backbones correspond to an anchor layer. This leads to scenarios $T^{*}<T_{\mathrm{bi}}^{*}$ for high and $T^{* *} \sim T_{\mathrm{bi}}^{* *}$ for low grafting densities. This theory was evaluated using PF6 and atactic PF1-8. Experiments show that $T^{* *}(\mathrm{PF} 6) \sim T_{\mathrm{bi}}^{* *}(\mathrm{PF} 1-8)$, which agrees with the low grafting scenario. The phase behavioral study was complemented by structural work. For both polymers, the structure of alternating backbone and side-chain layers was found rigid and similar on the $b c$ plane. This indicates that the backbone layer mimics the anchor layer of bidisperse block copolymer brushes and allows comparison across alkyl-substituted PFs with increasing $N$. PF6 side chains coming from opposite backbone layers were found segregated with the layer thickness of $H(\mathrm{PF} 6) \approx 5.6 \AA$ whereas the PF1-8 side chains are mixed in a bilayer with the half thickness of $H_{\text {bilayer }}(\mathrm{PF} 1-8) / 2 \approx$ $5.2 \AA$. For PF6 both grafting density regimes are possible $\left[b(\mathrm{PF} 6) \approx R_{c}(\mathrm{PF} 6) \approx H(\mathrm{PF} 6)\right]$, whereas $\mathrm{PF} 1-8$ represents the low grafting regime $\left[b(\mathrm{PF} 1-8)>R_{c}(\mathrm{PF} 1-8)\right]$. Forthcoming studies should expand these ideas to isotactic PF1-8 and from the solid state layers to sheetlike solution assemblies known for polyfluorenes [36] and other conjugated polymers, such as polyphenylenevinylenes [37].

\section{ACKNOWLEDGMENTS}

The research leading to these results has received funding from the European Community's Seventh Framework Program (Program No. FP7/2007-2013) CALIPSO under Grant Agreement No. 312284. M.K. acknowledges the Danish Agency for Science, Technology and Innovation via DANSCATT. 
[1] M. Ballauff, Macromolecules 19, 1366 (1986).

[2] S. B. Damman, F. P. M. Mercx, and C. M. Kootwijk-Damman, Polymer 34, 1891 (1993).

[3] J. J. Ge, A. Zhang, K. W. McCreight, R.-M. Ho, S.-Y. Wang, X. Jin, F. W. Harris, and S. Z. D. Cheng, Macromolecules 30, 6498 (1997).

[4] R. Stepanyan, A. Subbotin, M. Knaapila, O. Ikkala, and G. ten Brinke, Macromolecules 36, 3758 (2003).

[5] D. L. Cheung and A. Troisi, Phys. Chem. Chem. Phys. 11, 2105 (2009).

[6] M. J. Winokur, D. Spiegel, Y. Kim, S. Hotta, and A. J. Heeger, Synth. Met. 28, 419 (1989).

[7] K. Tashiro, K. Ono, Y. Minagawa, M. Kobayashi, T. Kawai, and K. Yoshino, J. Polym. Sci., Part B: Polym. Phys. 29, 1223 (1991).

[8] M. Grell, D. D. C. Bradley, G. Ungar, J. Hill, and K. S. Whitehead, Macromolecules 32, 5810 (1999).

[9] S. H. Chen, A. C. Su, C. H. Su, and S. A. Chen, Macromolecules 38, 379 (2005).

[10] M. Brinkmann, N. Charoenthai, R. Traiphol, P. Piyakulawat, J. Wlosnewski, and U. Asawapirom, Macromolecules 42, 8298 (2009).

[11] C. Liu, Q. Wang, H. Tian, Y. Geng, and D. Yan, Polymer 54, 2459 (2013).

[12] C. Liu, Q. Wang, H. Tian, J. Liu, Y. Geng, and D. Yan, Macromolecules 46, 3025 (2013).

[13] D. O'Carroll, D. Iacopino, A. O'Riordan, P. Lovera, E. O'Connor, G. A. O'Brien, and G. Redmond, Adv. Mater. 20, 42 (2008).

[14] J. Gao, M. A. Loi, E. J. F. de Carvalho, and M. C. dos Santos, ACS Nano 5, 3993 (2011).

[15] C. L. Donley, J. Zaumseil, J. W. Andreasen, M. M. Nielsen, H. Sirringhaus, R. H. Friend, and J.-S. Kim, J. Am. Chem. Soc. 127, 12890 (2005).

[16] M. Surin, P. Sonar, A. C. Grimsdale, K. Müllen, R. Lazzaroni, and P. Leclère, Adv. Funct. Mater. 15, 1426 (2005).

[17] M. Knaapila, D. W. Bright, B. S. Nehls, V. M. Garamus, L. Almásy, R. Schweins, U. Scherf, and A. P. Monkman, Macromolecules 44, 6453 (2011).

[18] H. Ozawa, T. Fujigaya, Y. Niidome, N. Hotta, M. Fujiki, and N. Nakashima, J. Am. Chem. Soc. 133, 2651 (2011).
[19] C. J. Kudla, N. Koenen, W. Pisula, and U. Scherf, Macromolecules 42, 3483 (2009).

[20] S. P. Schiess, N. Fröhlich, M. Held, F. Gannott, M. Schweiger, M. Forster, U. Scherf, and J. Zaumseil, ACS Appl. Mater. Interfaces 7, 682 (2015).

[21] T. M. Birshtein, Y. V. Liatskaya, and E. B. Zhulina, Polymer 31, 2185 (1990).

[22] R. J. Spontak, Macromolecules 27, 6363 (1994).

[23] H. Chen and A. Chakrabarti, Phys. Rev. E 52, 3915 (1995).

[24] R. Levicky, N. Koneripalli, M. Tirrell, and S. K. Satija, Macromolecules 31, 2616 (1998).

[25] W. A. Goedel, C. Luap, R. Oeser, P. Lang, C. Braun, and R. Steitz, Macromolecules 32, 7599 (1999).

[26] G. Kritikos and A. F. Terzis, Polymer 46, 8355 (2005).

[27] J. Burdynska, W. Daniel, Y. Li, B. Robertson, S. S. Sheiko, and K. Matyjaszewski, Macromolecules 48, 4813 (2015).

[28] S. Minko, I. Luzinov, V. Luchnikov, M. Müller, S. Patil, and M. Stamm, Macromolecules 36, 7268 (2003).

[29] D. Romeis and J.-U. Sommer, ACS Appl. Mater. Interfaces 7, 12496 (2015).

[30] W. M. de Vos and F. A. M. Leermakers, Polymer 50, 305 (2009).

[31] M. Knaapila, R. Stepanyan, M. Torkkeli, B. P. Lyons, T. P. Ikonen, L. Almásy, J. P. Foreman, R. Serimaa, R. Güntner, U. Scherf, and A. P. Monkman, Phys. Rev. E 71, 041802 (2005).

[32] M. Grell, W. Knoll, D. Lupo, A. Meisel, T. Miteva, D. Neher, H.-G. Nothofer, U. Scherf, and A. Yasuda, Adv. Mater. 11, 671 (1999).

[33] N. Fröhlich, Isotaktische Polyfluorene - Synthese, Isolierung und Charakterisierung, Ph.D. thesis, Bergische Universität Wuppertal, 2014.

[34] S. H. Chen, A. C. Su, C. H. Su, and S. A. Chen, J. Phys. Chem. B 110, 4007 (2006).

[35] M. Torkkeli, F. Galbrecht, U. Scherf, and M. Knaapila, Macromolecules 48, 5244 (2015).

[36] M. Knaapila, D. W. Bright, R. Stepanyan, M. Torkkeli, L. Almásy, R. Schweins, U. Vainio, E. Preis, F. Galbrecht, U. Scherf, and A. P. Monkman, Phys. Rev. E 83, 051803 (2011).

[37] W.-C. Ou-Yang, C.-S. Chang, H.-L. Chen, C.-S. Tsao, K.-Y. Peng, S.-A. Chen, and C. C. Han, Phys. Rev. E 72, 031802 (2005). 